\title{
Carnets
}

Revue électronique d'études françaises de l'APEF

Deuxième série - $18 \mid 2020$

Chiens et écritures (littéraires, filmiques, photographiques)

\section{Les chiens du rêve américain : représentations, récits et les limites de l'anthropomorphisme dans le cinéma hollywoodien et indépendant}

\section{Argyrios Keleris}

\section{(2) OpenEdition}

\section{Journals}

Édition électronique

URL : https://journals.openedition.org/carnets/10951

DOI : 10.4000/carnets. 10951

ISSN : 1646-7698

Éditeur

APEF

Référence électronique

Argyrios Keleris, «Les chiens du rêve américain : représentations, récits et les limites de

l'anthropomorphisme dans le cinéma hollywoodien et indépendant », Carnets [En ligne], Deuxième série - 18 | 2020, mis en ligne le 31 janvier 2020, consulté le 21 septembre 2021. URL : http:// journals.openedition.org/carnets/10951 ; DOI : https://doi.org/10.4000/carnets.10951

Ce document a été généré automatiquement le 21 septembre 2021.

\section{(c) (7) (9)}

Carnets est mis à disposition selon les termes de la licence Creative Commons - Atribution - Pas d'utilisation commerciale 4.0 International. 


\title{
Les chiens du rêve américain : représentations, récits et les limites de l'anthropomorphisme dans le cinéma hollywoodien et indépendant
}

\author{
Argyrios Keleris
}

\section{Introduction}

1 La représentation de plus en plus marquée de l'animal canin dans les arts et les médias populaires, comme le cinéma, la littérature et les «textes» numériques, est indissociable d'une explosion récente de la recherche consacrée à l'observation de son « comportement » dans les différents types de ses relations avec l'animal humain. Si le chien est aujourd'hui devenu objet d'étude des sciences telles que l'éthologie cognitive et les animal studies, cela est dû à deux principales raisons : d'une part, au fait que l'être humain n'apparaît plus comme l'unique sujet de la signification et du sens, à l'intérieur d'un discours qui n'est plus exclusivement affaire ni délimité au service de l'humanité ; et, d'autre part, au fait que nos attitudes envers les chiens ont subi, elles aussi, un changement radical, qui tente de libérer ces animaux de leurs statuts anciens comme animaux de chasse, ou comme outils à l'usage des humains dans l'agriculture et l'élevage, pour en faire une espèce de compagnie, " éléments de la culture humaine " (McLean, 2014: 12), leur valant le surnom du «meilleur ami de l'Homme». Or le compagnonnage homme-canidé peut-il être porteur pour et en lui-même d'une signification autre que celle que les théories culturalistes tentent de lui attribuer? N'impliquerait-il, en d'autres termes, un sens et une valeur qui suffiraient à euxmêmes, qui seraient alors identiques à la relation elle-même, et comment pourrait-on décrire cette relation en ces termes, c'est-à-dire au-delà des associations symboliques et mythiques qu'elle mobilise? Cet article propose une étude des différents procédés 
que le cinéma américain a employés pour poser la question de notre relation avec l'animal canin, et de la manière dont ceux-ci peuvent être ramenés à une opposition fondamentale entre, d'une part, une approche utilitariste et anthropomorphique de cette relation, inséparable d'une certaine politique de ce cinéma à l'égard du récit et de la représentation, et, d'autre part, une redéfinition de cette relation, comme quelque chose qui se passe entre les termes qu'elle relie, à savoir l'homme et le chien, sans primat de l'un sur l'autre.

\section{Les chiens du cinéma hollywoodien : la machine anthropologique de la narration}

Dans la plus grande partie du cinéma hollywoodien, l'«économie» des relations homme-chien ne peut s'édifier qu'en prenant le chien comme moyen de négociation et de résolution de conflits proprement humains. Là, un type de «machine anthropologique » est à l'œuvre, dans le sens d'un jeu productif entre l'humanisation du chien et l'animalisation de l'homme à l'issue duquel une histoire ou une identité en crise retrouve et affirme son ancien point d'équilibre, ou en construit un nouveau. Nous verrons pourquoi la représentation du chien dans ce cinéma est inextricablement liée à un modèle narratif auquel elle doit s'adapter. Ce modèle, que des narratologues comme David Bordwell ont analysé sous le terme de classicisme cinématographique, a trouvé dans le cinéma hollywoodien l'expression la plus vive et la plus achevée à travers une série de caractéristiques qui sont restées au fil des années relativement stables (Bordwell, 2006 : 35). La caractéristique principale du cinéma hollywoodien réside dans sa manière particulière d'illustrer la capacité d'un héros à évoluer par le biais d'actions conscientes qui le conduisent à la réalisation d'un but clairement déterminé. Les obstacles que le héros se doit de surmonter pour pouvoir évoluer reflètent le plus souvent les contradictions sociales et culturelles qui sont inhérentes à la société américaine, et pour lesquelles le classicisme offre les stratégies idéologiques de négociation et de résolution (Schatz, 1981: 30). Pour Gilles Deleuze le récit hollywoodien typique impliqué dans le régime de «l'image-action» (Deleuze, 1983 : 210) porte sur un héros individuel ou collectif qui intervient par son action entre deux ou plusieurs systèmes de valeurs opposés (contractées au sein d'une situation) pour mettre fin à leur conflit, soit par l'élimination de l'un de ces systèmes (et le triomphe de l'autre), soit par leur pacification dans des formules de compromis. Ces systèmes de valeurs ne sont autres que les constituants, par nature contradictoires, du rêve américain, et la résolution de leur conflit est le plus souvent ritualisée dans une séquence finale qui célèbre le caractère intemporel et stable de l'identité américaine.

Comme tout animal filmique, si l'on s'en tient à l'idée centrale de Jonathan Burt, le chien « est pris dans un espace incertain entre le naturel et l'artificiel» (Burt, 2002 : 10), et cela parce qu'il est doté par la nature, au même titre que l'animal humain, lui aussi perçu dans sa matérialité pure en-deçà du langage, d'émotions et de sensibilité. C'est par là que, pour Burt, il peut devenir vecteur d'une valeur morale et sociale, avant l'instrumentalisation de cette dernière au niveau narratif et représentatif. Cette capacité constitue le «potentiel moral» (Burt, 2002: 23) du chien filmique, qui s'incarne d'autant mieux lorsque ce dernier croise son regard avec celui d'un personnage humain, lorsqu'il lui adresse comme une attente désintéressée ou un respect «sans compréhension». Pour Adrienne McLean, les chiens filmiques 
provoquent une "crise de représentation", non seulement parce qu'ils affectent la manière dont on les perçoit dans la vie réelle, mais aussi parce que leur représentation ne peut pas être pleinement perçue comme telle. Un film de fiction impliquant un chien est toujours « documentaire » dans un certain sens, et l'oscillation entre les contextes représentatifs, entre les chiens comme événements et comme fiction, peut avoir des conséquences profondes (McLean, $2014: 20$ ).

4 À l'autre bout de cette croyance à ce que l'animal exemplaire peut incarner comme par nature dans sa représentation filmique, de manière à pouvoir finalement lui résister, se trouve l'idée partagée par des théoriciens comme John Berger (Berger, 1980) et Akira Mizuta Lippit (Lippit, 2000), selon laquelle, dès qu'on réduit l'animal à un signe, ou comme Lippit l'exprime, à une "spectralité » (Lippit, 2000:1), on s'éloigne de l'expérience directe de l'animalité en l'animal et en nous-mêmes, qui supposerait un rapport pratique non représentant, c'est-à-dire sans médiation par l'image, rapport qui caractérisait les sociétés préindustrielles (Berger, 1980: 2-5). Ces types d'argumentations d'après lesquelles les animaux disparaissent dans une culture fondée sur le regard, qui réduit l'animal au statut de signe par l'instauration entre lui et l'être humain des lignes de séparation, nous conduisent au problème de l'anthropomorphisme qui se trouve au cœur d'une controverse animant le champ des animal studies.

5 En matière de cinéma, l'anthropomorphisme concerne aussi bien le stéréotype de l'image, à savoir l'attribution directe à l'animal écranique de valeurs morales liées à une certaine époque et déterminant pour l'animal un statut social profilmique, que le stéréotype du récit, à savoir l'héroïsation de l'animal ou son emploi comme médiateur de conflits interhumains. Pour ce qui est de la représentation directe des chiens, nous savons aujourd'hui la fréquence avec laquelle le cinéma hollywoodien n'a de cesse d'en faire des humains peluchés, portant des costumes et parlant de voix doublées, notamment depuis les « All Barkie Dogville Comedies » de MGM produits entre 1929 et 1931, et « initialement » conçus pour parodier les genres de cette époque. C'est ainsi, en plus, que Kelly Wolf examine les processus de mythologisation de Lassie dans les années 1940 et 1950 comme l'exemplaire du chien idéal - loyal, fort, intelligent et protecteur - conformément aux besoins sociaux d'idéalisme et d'héroïsme en des temps de guerre, ainsi que de "pureté de race et de genre " (Wolf, 2014:22). Propulsés au rang de star, aux côtés de Lassie, d'autres chiens, notamment des bergers Allemands, de Strongheart et Rintintin à Jerry Lee (de la saga contemporaine Chien de flic) peuplent un cinéma qui, dans la tradition des films des premières années après la guerre, continue jusqu'à nos jours à humaniser des types de chiens en les « dotant à la fois, à travers des récits héroïques, de facultés intellectuelles complexes et du courage » (Fuller-Seeley, Groskopf, 2014 : 56). Nous pouvons citer, à titre d'exemple, les franchises commerciales comme Doctor Dolittle $(1998,2001)$, Beverly Hills Chihuahua $(2008,2011,2012)$, et Cats and Dogs (2001, 2010), ainsi que les « buddy films » de Disney, comme Air Bud: Buddy star des paniers de 1997, dans lesquels une série de chiots parlants passent leurs talents pour les sports à des enfants solitaires et timides, les aidant souvent à emporter des tournois de leurs écoles.

6 Ainsi, pour ce qui est de la représentation indirecte, que celle-ci soit perçue comme un compromis du désintéressement et de la "compréhension au-delà du langage » impliqués dans le "potentiel moral» de la nature canine, ou comme une élaboration supplémentaire du stéréotype dans l'image, le rôle du medium filmique a été de 
nouveau décisif. Kathryn Fuller-Seeley et Jeremy Groskopf voient, par exemple, dans l'intégration de l'animal canin au récit, par où il devient capable d'offrir des solutions aux problèmes des hommes (par exemple de sauver un membre de la famille humaine ou de l'aider à surmonter la perte d'un autre), et pour lesquelles il est récompensé, félicité par des caresses, des embrassements et des encouragements verbaux, un processus de récupération de sa nature par les humains (Fuller-Seeley, Groskopf, 2014 : 65). Ainsi, la compréhension mutuelle et partagée entre l'homme et le chien se restaure en même temps qu'une identité de leur action, à l'intérieur d'un univers d'échanges intéressés, régi par un principe que l'on pourrait appeler «narcissisme canidophilique ", d'après l'analyse de Donna Haraway (Haraway, 2003: 33). Par conséquent, des émotions perçues comme naturelles chez les humains deviennent non naturelles par le biais du récit lorsqu'elles sont appliquées aux animaux.

De ce qui précède nous pouvons comprendre que théoriser l'emploi du chien dans un film suppose de penser sa relation au personnage humain, comme entre la nature et la culture, l'ouverture de son potentiel animal et la fermeture de sa stéréotypisation narrative, en tant qu'une relation proprement agonistique. Il n'est pas, à cet égard, un hasard que le personnage canin dans plusieurs films hollywoodiens des années 1920 était introduit comme appartenant à une race croisée, ou comme moitié loup moitié domestiqué, afin que le récit se concentre sur son pouvoir de mettre sa nature sauvage au service des humains, ou la dénier dans un nouveau rôle de chien de compagnie. Pour Susan McHugh, plusieurs personnages canins occupent narrativement des espaces liminaires, et comme Cerbères, le gardien mythologique des portes entre les Morts et les Vivants, constituent des liens tantôt d'opposition tantôt de conciliation entre différentes valeurs humaines (McHugh, $2004: 41$ ).

Le récit de Fidèle Vagabond (Old Yeller, Robert Stevenson-1957), produit par Disney est paradigmatique de cette conception du personnage canin comme médiateur. Travis est un garçon qui, après le départ de son père pour escorter du bétail, se voit confié la garde de la famille. Lorsqu'un labrador errant est recueilli par Arliss, son frère cadet, Travis est d'abord méfiant et tente de le chasser. Mais quand le chien sauvera la vie d'Arliss des griffes d'un ours, Travis décidera de l'adopter pour devenir progressivement son meilleur ami. Vers la fin du film, le chien est touché par la rage, et Travis le tue. Cette évolution, bien qu'inhabituelle pour un film de Disney, est un exemple de tension entre un anthropomorphisme narratif tout-puissant et la capacité naturelle de l'animal de résister aussi bien à son illustration sentimentale qu'à son instrumentalisation comme moyen narratif de négociation de conflits proprement interhumains. À l'égard de ce qui nous intéresse ici, il faut pourtant s'interroger si la disparition de l'animal de la ligne explicite narrative suffirait pour menacer l'ordre symbolique instauré par cette ligne. Pour appliquer à notre cas le concept de "transgression constitutive" analysé par Slavoj Žižek au sujet de la femme fatale comme élément d'altérité dans les crime films du classicisme américain (Žižek, 2005 : 215-217), la survivance de l' " image spectrale » du chien, à travers les émotions que son statut matériel provoque aux spectateurs au-delà de l'intrigue, loin d'être une menace effective à la loi symbolique, constitue son propre support dans un film hollywoodien d' « image-action » comme celui-ci. La disparition de l'animal signifierait alors que si le passage à l'âge adulte d'un garçon, et la restitution de l'harmonie familiale, ne peuvent pas avoir lieu sans blessures, celles-ci ne doivent en aucun cas les entraver (Burt, 2002 : 181). À titre indicatif, le fait que Lisbeth, amie-voisine de Travis offre à ce dernier son chiot, né du mariage entre Vagabond et sa chienne, Miss Priss, suggère que les deux 
adolescents eux-mêmes projettent de se marier (Brode, $2004: 190)$, faisant du chien le représentant de leur désir et la marque d'une alliance à venir.

On pourrait dire que le chien filmique des récits hollywoodiens constitue l'analogon du Westerner, personnage médiateur entre la sauvagerie et la civilisation dans les westerns classiques. Il faut analyser en ce sens la forme d'apparition du chien dans le film de Stevenson. Personnage par excellence du milieu, à la fois tendre et agressif, héroïque et inapprivoisable, Vagabond, comme bon nombre de ses homologues humains du western, apparait dans la séquence d'ouverture $d u$ film alternant plans fixes et travellings, moments de repos et de mouvement, sur le chemin qui le mènera de cet espace intermédiaire, symbolisant le monde sauvage de la nature, comme laissent entendre les plans qui le montrent en train de chasser des lapins, à celui d'une famille humaine. Cependant, si les forces de l'anomie, que le héros traditionnel du western doit vaincre pour restaurer l'ordre social, sont le plus souvent le résultat des actions de personnages individualistes, délinquants, d'Indiens, etc., dans Fidèle Vagabond elles représentent directement le côté sauvage, anarchique, insensé de la nature. Ainsi, le chien prouvera son héroïsme et sa dévotion, en faisant successivement face à un ours, une horde de cochons fous et un loup, qui menacent la propriété familiale et la vie des enfants. Et s'il est finalement conduit hors du récit, à l'image - ou presque - des Westerners humains (n'ayant jamais réellement intégré le monde de la civilisation, ils disparaissent soit en mourant soit en repartant à leur lieu naturel, derrière les montagnes, dans le désert), ce n'est que parce qu'il a rejoint définitivement le monde de l'autre côté qu'il a combattu, en développant de la rage à la suite de sa morsure par le loup. En prenant symboliquement le même chemin du retour que les héros humains du western, le chien aura toutefois accompli sa fonction mythique ${ }^{1}$. C'est à cet égard que la scène finale dans laquelle le nouveau chien adopté par la famille est en train de chasser des lapins, ne s'éloigne pas des conventions génériques d'épanadiplose, dans le sens qu'elle reprend quasi identiquement le motif du début, afin de mettre en valeur une certaine idée signifiante ou de construction. D'après celle-ci la vie en famille restreinte (éthos : habitat) et élargie (ethnos : nation) est un cycle que les éléments anomiques de maladie, de violence et de mort ne peuvent pas interrompre. En effet, Sam l'intrépide (Savage Sam, Norman Tokar - 1963) constitue une reprise de ce cycle, retrouvant quelques années plus tard les mêmes personnages de Travis, Arliss et Lisbeth, et leur chien Sam qui part sur leurs traces quand ils sont enlevés par des Indiens. Repérant des analogies thématiques et iconographiques entre le film de Disney et le western classique La Prisonnière du Désert (The Searchers, John Ford - 1956), Douglas Brode trouve dans le personnage de Beck, oncle de Travis, l'équivalent d'Ethan, interprété par John Wayne dans le film de Ford (Brode, 2004 : 197). Cependant, sans la vaillance et le vif sens de l'odorat de Sam, l'équipe de recherche formée par quelques vétérans du genre, comme slim Pickens et Royal Dano, n'aurait pas été capable de retrouver les enfants.

Médiateur de conflits proprement interhumains, le héros canin l'est aussi bien dans le cadre d'un autre genre populaire, celui du hardboiled detective film. Nous voyons là une évolution dans la convention du chien partenaire des humains, qui le conduit loin de la frontière et des milieux ruraux, dans le contexte anomique plus diffus et moins clairement délimité des grandes villes, où il s'engage aux côtés des adultes au service de la loi. Cette évolution dans le compagnonnage filmique entre l'homme et le chien, est le résultat, si l'on accepte l'explication fournie par Tim Gadd (Gadd, 2005 : 250-251), d'un processus d'approfondissement de la domestication et de la socialisation de l'animal, 
par lequel ce dernier s'est progressivement éloigné des enfants (avec qui il se liait sur la base de "conditions pré-linguistiques" communes) pour pénétrer dans les affaires émotionnelles et sociales des adultes. Si cette évolution est indicative d'une nouvelle réalité dans l'époque postmoderne, où aux théories anthropocentriques traditionnelles s'est ajoutée une tendance qui veut que la distinction entre les humains et les animaux devienne de plus en plus vague, et où finalement comme McHugh soutient, le rapprochement homme-animal dans les arts représentatifs va de pair avec un besoin de séparer les deux termes selon leurs propres moyens de mobiliser les processus de représentation ou de les arrêter (McHugh, 2011 : 11-12), il est tout aussi vrai que, en dernière analyse, ces moyens ne peuvent pas échapper à leur subordination finale à la narration. Cela concerne aussi le plus souvent les genres de thriller et de science-fiction où l'attribution aux chiens de puissances diaboliques, bien que fidèle à leur " capacité » naturelle, en tant que créatures liminaires, de déstabiliser notre raison historiquement située, n'est pas sans rapport avec un discours scientifique et médiatique qui les réinstaure narrativement comme objets, machines, et produits de notre connaissance (Molloy, $2011: 9$ ).

\section{Les chiens du cinéma américain mineur : mort et solitude aux creux du récit}

11 Pour Deleuze, à l'opposé de la grande forme hollywoodienne de l'image-action et du « cinéma d'actant» se trouve un «cinéma de voyant» (Deleuze, 1985: 166), ou un " cinéma mineur» (Deleuze, 1985 : 282-291), qui prend acte du fait que le «sousdéveloppement » des minorités, leur impuissance à accéder à une « langue » majeure, doit se refléter en matière filmique comme stagnation du récit et comme écroulement du régime représentatif. La stagnation du récit ou l'absence de progression narrative signifie que les termes traditionnellement opposés dans le cinéma hollywoodien, n'arrivent pas à une conciliation, où à une négociation dialectique qui les nierait, mais se donnent à voir en tant que différences, dans les interstices et les brèches qu'ils ouvrent dans le récit. $\mathrm{Si}$, au niveau narratif, la stagnation filmique suppose des personnages minoritaires qui sont incapables d'agir sur leurs conditions afin de les changer, au niveau esthétique cette même impuissance se traduit, suivant Deleuze, en une puissance de contemplation d'un monde libéré du sens et de la signification, et donc affirmé dans toutes ses différences préservées comme "pures situations optiques et sonores " (Deleuze, 1985 : 356). Dans ces mêmes conditions, quand rencontre il y a entre des personnages minoritaires, elle ne parvient pas à réaliser une promesse d'un monde autre, réel ou idéal, parce que celle-ci supposerait un peuple déjà-là. $A u$ contraire, dans le cinéma mineur, le peuple manque (Deleuze, 1985 : 288), car il est sans cesse obligé de s'inventer et de se réinventer à travers ses expériences d'actions nonaccomplies ou de non-actions ${ }^{2}$. Dans le cinéma américain, le problème de l'expression minoritaire en ces termes se pose de manière particulièrement intense, puisque dans ce pays de contradictions on a affaire non seulement avec un "rêve américain ", «la promesse que tous les Américains ont une chance raisonnable (...) d'atteindre la vertu et la pleine réalisation de soi par leur succès » (Hochschild, 1995: xi), mais aussi avec une réalité qui rend cette promesse inaccessible pour de larges tranches de la population. Ce qui est remarquable dans certains films du cinéma américain mineur, c'est que l'enjeu de la constitution d'un peuple ou d'une communauté, se pose aussi 
bien entre les personnages humains et les personnages canins, qu'entre un personnage humain et un autre. Et il se pose d'autant plus puissamment qu'il reste, à l'issue des rencontres empêchés et de réciprocités avortés, entre l'homme et le chien, un enjeu fondamentalement ouvert.

Dans Le teckel (Wiener-Dog, Todd Solondz-2016), un garçon souffrant de cancer reçoit en cadeau une chienne qu'il nomme Wiener-Dog. Semant le trouble dans la vie de la famille, Wiener-Dog se fera stériliser, avant d'être confiée à un vétérinaire pour qu'il s'occupe de son euthanasie. Sauvée par une jeune infirmière, elle commencera alors un long voyage à travers l'Amérique du Nord, passera dans les mains d'un couple de trisomiques, d'un cinéaste sans inspiration, et d'une femme dépressive, se fera écraser par une voiture, pour ensuite se transformer en objet d'art exposé en musée, par un spécialiste de l'animation électronique. Pendant toute cette chronique, elle deviendra témoin de la solitude et de la tristesse des autres - monument vivant de sa propre disparition, si nous voulons revenir à l'idée centrale de Berger, ainsi que de celle des hommes. La question de l'anthropomorphisme est traitée par Solondz de manière assez caractéristique dans la scène où une mère (Julie Delpy) tente d'expliquer à son fils les avantages de l'hystérectomie en lui racontant l'histoire de sa propre chienne en France, une caniche "drôle et gentille ", qui a été « violée " par un chien errant dans les bois, "griffé, graisseux et laid ». Préoccupé par le problème du stéréotype et de la manière dont un récit filmique le présuppose, y compris par le biais de l'onomatologie (déjà dans Palindromes de 2004 des prénoms palindromiques comme Aviva et Bob s'ajoutent à la forte stéréotypisation gestuelle et comportementale des personnages), il ne faut pas s'étonner que Solondz ait choisi de nommer la chienne violée "Croissant ", et le chien violeur « Mohammed».

Bien que l'emploi filmique de Wiener-Dog, comme animal de compagnie d'une constellation entière d'hommes et de femmes marginaux, soit indicateur d'un phénomène émergeant dans une société occidentale de plus en plus multiculturelle, que Gadd appelle " égalitarisme d'espèces » (Gadd, $2005: 257)$, il est tout aussi vrai que la rencontre de ces personnages avec la chienne n'arrive pas à l'équilibre harmonieux tant promu par la progression narrative des textes hollywoodiens. Ainsi, à l'encontre de ce à quoi le cinéma hollywoodien a excellé en instrumentalisant les produits des fonctions naturelles de l'animal canin, comme son aboiement dans les comédies screwball, pour mettre en valeur «la flexibilité du statut social de ses compagnons humains » (Ross, Castonguay, 2014: 91), ou en les éclipsant totalement, comme les excréments, jamais illustrés, par exemple, dans les films de Lassie (McHugh, $2004: 109$ ), l'intérêt que porte Solondz à son personnage animal comme une créature exposée, au même titre que les personnages humains, à la vulnérabilité et la finitude du monde de la matière, l'amènera à des solutions différentes. D'une part, la relation affective qui était en train de s'établir entre ces deux créatures, l'enfant et l'animal, unies dans leurs maladies et leurs solitudes respectives, sera interrompue violemment par les parents, qui décident d'euthanasier Wiener-Dog. D'autre part, le cinéaste n'hésitera pas de filmer, d'abord par un long travelling, une trainée d'excréments laissés par la chienne après une diarrhée, et plus tard, par un plan fixe, distancié, son sang écoulé de sa cage après l'ablation de son utérus. Dans la deuxième partie du film, on retrouvera WienerDog aux côtés de deux autres personnages solitaires, la vétérinaire qui l'a sauvée de l'euthanasie et son ami toxicomane. Dans une scène caractéristique de l'approche solondzienne de l'animal comme opérateur de liens impossibles avec des hommes 
socialement exclus et impuissants, à l'intérieur sombre d'une chambre de motel, la caméra balaie en panoramique qui réunit le chien avec le couple et trois musiciens clandestins croisés en route, en tant que créatures séparées, dans cet abîme où il devient possible de les penser comme un peuple qui manque.

En outre, la stérilité de la chienne trahit une homologie structurale avec celle de sa troisième détentrice dans le film, une femme trisomique (April) qui, comme son beaufrère confie à son amie, ne peut pas avoir des enfants, car elle s'est fait « les trompes ligaturées ». Le commentaire introduit ici par Solondz a plusieurs fonctions : d'une part, de rapprocher la chienne avec une partie de la population socialement et génétiquement impuissante (les personnes trisomiques) et, d'autre part, de soulever la question de l'eugénisme de manière à opposer sa Wiener-Dog à tous les chiens vedettes qui ont peuplé le cinéma hollywoodien, de Rintintin et Strongheart à Lassie, dont l'image l'industrie hollywoodienne avait tout intérêt de pérenniser, au-delà de leur mort biologique, conformément à ses exigences de reproduction et de stabilité (Wolf, 2014 : 117-119). D'ailleurs, ce qui est vrai pour la rencontre entre April et Wiener-Dog, peut s'appliquer métonymiquement sur l'ensemble du corps social dépeint par Solondz comme stérile, à savoir comme incapable pour le nouveau et le changement. Après son écrasement par un camion qui provoque sa mort, Wiener-Dog revient mécaniquement à la vie par les mains d'un artiste qui la transforme en une animatronique, l'empaillant et la robotisant pour l'exposer dans une galerie d'art. Le chien qui nous regarde et nous aboie à la fin du film réalise-t-il le rêve trop humain d'un amour inconditionnel de l'animal, et d'attribution à celui-ci d'un langage qui le rend capable (via la technologie) de mourir, en le restituant à la vie ? Quoi qu'il en est, le cinéaste par ce dernier geste nous réinvite à la problématique qui lui est chère, celle d'une société qui (re)devient " nature » au terme d'un processus de totalisation et d'hyperréalisation par lequel, à force de déterminer si radicalement les êtres, elle finit par se dissocier et se désocialiser elle-même (Baudrillard, 1990 : 119-128).

Le scénario de Fruitvale Station (Ryan Coogler, 2014) est tiré d'un tragique fait divers. Il raconte les 24 heures qui ont précédé le meurtre par la police d'Oscar Grant, un jeune père de famille noir. Dans une scène de ce film dont le récit se suspend au milieu des vas-et-viens quelconques et incertains d'Oscar, un chien s'introduit à la vie de ce dernier aux environs d'une station-service désertique, pour mourir aussitôt écrasé par une voiture. Sa mort dans les bras d'Oscar n'est pas simplement un signe prémonitoire de sa propre mort, mais l'événement d'une rencontre impossible entre deux créatures homologues, qui partagent la fermeture d'être au monde - et, peut-être, aussi, l'ouverture infinie d'une promesse, d'un peuple qui manque. Dans le film Collateral (Michael Mann, 2004) quatre autres créatures se lient et se séparent définitivement autour d'une promesse qui ouvre une brèche à l'intérieur d'un récit déployant toute l'iconographie du crime film hollywoodien, bien que d'une manière particulière qui l'éloigne des conventions idéologiques du genre. Vincent, un tueur à gage, dépeint, au moins dans un premier temps, comme une personne "de supériorité amorale et athéistique » (Rayner, 2013: 82) contraint Max, un humble chauffeur de taxi, de l'aider à accomplir une mission immobilière. Vers le milieu de celle-ci et pendant que Max conduit son taxi avec Vincent sur le siège arrière, deux coyotes apparaissent soudainement devant eux. Max ralentit et le film tombe dans un temps mort, fait des regards croisés entre ces animaux sauvages de l'espèce de canidés, et les personnages. Dans la musique de fosse, Chris Cornell chante "I can tell you why people die alone", pendant que les deux personnages reprennent silencieux, le regard dans le vide, leur 
trajet. Les canidés, dépossédés de leur territoire et faisant valoir celui, hyper-urbain, dans lequel Max et Vincent errent, comme un autre désert, apparaissent comme des analyseurs sauvages de la futilité des actions des personnages et de l'impuissance de ces derniers à se retrouver par le biais du langage. En tant qu'expérience de l'altérité, la rencontre avec ces animaux ouvre aussi pour les protagonistes (et les spectateurs) l'espace nécessaire pour penser leur « être » comme mortel, et donc, comme un « être de l'autre ». Pour le dire comme Anat Pick, de cette brèche ouverte au milieu de l'image-action, la mort surgit comme « la contingence absolue [qui] marque la limite de la représentation, en capturant une réalité qui fuit la représentation » (Pick, 2011 : 113).

Wendy et Lucy (Kelly Reichardt, 2008) est un film américain mineur dans lequel le chien apparaît comme personnage à part entière aux côtés d'une jeune femme chômeuse en plein voyage pour Alaska où elle espère trouver un emploi dans le traitement des saumons. Un long travelling latéral introduit les deux amies, Wendy et Lucy, qui jouent va chercher dans la clairière d'une forêt, alors que la première fredonne une triste mélodie qui plonge la scène dans une ambiance d'inquiétude. Cette première scène sert d'intrigue de prédestination à ce récit oscillant entre la tendresse immanente au lien homme-chien et l'angoisse de voir ce dernier compromis par une réalité sociale et économique particulièrement défavorable. Lorsque Lucy se joint à un groupe de jeunes squatteurs assis autour d'un feu de camp qui souligne sur leurs visages la fatigue et le désœuvrement, un homme fait apprendre à Wendy qu'il avait travaillé dans une pêcherie d'Alaska, avant d'être licencié pour avoir accidentellement détruit du matériel. Wendy, l'air réprobateur et agité, pressentant probablement que son propre avenir pourrait ressembler à celui de ces jeunes vagabonds, récupère sa chienne et reprend son chemin. Pourtant, une série d'événements va progressivement confirmer ses peurs. Sa voiture tombera en panne et, en attendant qu'elle soit réparée, elle se trouvera obligée de dormir dans la rue. En outre, arrêtée dans un supermarché pour avoir volé de la nourriture pour chien, elle passera une nuit au commissariat, avant de s'apercevoir, à sa sortie, que Lucy, son seul lien affectif avec le monde, a disparu.

17 L'intérêt croissant des films américains contemporains pour des personnages marginaux et leurs luttes pour survivre à l'ombre du rêve américain a conduit le critique A.O. Scott à forger le terme de "néo-néoréalisme américain » (Scott: 2009), pour souligner les affinités thématiques que ces films, parmi lesquels Wendy et Lucy, partagent avec le courant cinématographique apparu en Italie au lendemain de la seconde guerre mondiale. Pour Elena Gorfinkel, le film de Reichardt doit beaucoup à la tradition du néoréalisme italien et, plus particulièrement, aux films de Vittorio De Sica Le Voleur de Bicyclette (Ladri di biciclette, 1948) et Umberto D. (1952) (Gorfinkel, 2012 : 325). Cependant, si dans Le Voleur de Bicyclette la trame narrative est construite autour d'un objet comme la bicyclette, sa perte et la quête de Ricci pour le retrouver parce qu'il lui est indispensable pour son travail, dans Umberto D., comme dans Wendy et Lucy, l'utilité des "objets perdus", à savoir des chiens Flike et Lucy respectivement, est de nature plutôt émotionnelle que pratique. Lorsque Wendy se rend à la fourrière pour chercher Lucy, la caméra épouse son point de vue, en balayant, cage après cage - en décalage profond, donc, avec le travelling d'ouverture qui suivait le chien dans sa course en liberté - une série de chiens abandonnés, comme mutatis mutandis, dans le travelling subjectif de De Sica, Ricci cherchait vainement dans une rangée de vélos, à restaurer son dernier lien avec le monde ${ }^{3}$. Cela ne doit en aucun cas nous amener à considérer les liens émotionnels comme étant de moindre importance pour des gens dont le quotidien est dans sa plus grande partie occupé par la lutte pour la survie. Dans son étude 
comparative de Wendy et Lucy et du Voleur de Bicyclette et, tout en souscrivant à un point de vue, d'inspiration marxiste critique, selon lequel l'amour de Ricci pour son fils ne suffirait ni pour le nourrir ni pour le vêtir, Will Fech soutient en même temps que «dans des milieux ravagés par la précarité économique (...) le drame d'un chien perdu acquiert une nouvelle signification émotionnelle et symbolique. Quand la pauvreté est déjà là, d'autres manques et privations montent à la surface et assument un poids dramatique » (Fech, $2018:$ 108).

18 Lucy, depuis le moment où elle est mise en retrait du récit, hante ce dernier par son absence. C'est pourquoi Reichardt insiste tant à mettre en parallèle les sorts des deux personnages, ou à combiner leurs luttes pour la survie en une seule (Fech, $2018: 111$ ). Après l'incident de sa confrontation avec un sans-abri sinistre et psychotique au milieu d'une forêt où elle a campé la nuit, Wendy se précipite dans la salle de bains d'une station-service, et chuchote en pleurant d'effroi devant l'évier : " Accroche-toi, ma fille. J'arrive ». Ces propos deviennent source d'émotions pour les spectateurs, d'autant plus qu'ils sont adressés à Lucy, une autre créature seule, présumée perdue et apeurée dans un environnement dangereux, à la suite de deux scènes en contrepoint figurant le sommeil de Wendy avec et sans Lucy. Plus tôt dans le film, nous avons pu lire sur le visage de Wendy les marques de l'angoisse, alors qu'elle voyait le temps passer sur une horloge du commissariat, sachant que sa chienne l'attendait attachée à une colonne devant un supermarché. La scène où les policiers locaux l'obligent à deux reprises à donner ses empreintes digitales, fait alterner images informatiques de ces dernières, alors qu'elles sont prélevées sur une lame en verre, et plans rapprochés sur le visage inquiet de Wendy, habitué jusqu'à alors à un autre type de reconnaissance, inspirée à sa compagnonne canine, sa tendre moitié qu'elle ne voit plus. Commentant sur cette mise en parallèle de ces deux types d'identification, Dominic Pettman, écrit «que l'empreinte digitale a la fonction inverse, même si elle fixe l'identité unique de Wendy pour les besoins de l'identification officielle », la réduisant « à une vie nue, une donnée purement biologique »(Pettman, 2017 : 93).

19 À la fin du film, lorsque Wendy retrouve Lucy dans la cour arrière de sa nouvelle maison d'accueil, elle s'aperçoit que son amour pour sa chienne ne suffit pas pour la garder auprès d'elle. Sans moyen de transport et pratiquement sans argent, une loi toute-puissante au-dessus de ces deux créatures qui partageaient jusqu'alors le même but de survivre, est en train de les séparer. Wendy pleure à travers la clôture grillagée alors qu'elle annonce à Lucy sa décision de reprendre seule son voyage, plaçant l'amour pour sa chienne au-dessus de son besoin d'être aimée à son tour. Qu'elle soit consciente ou non de ce que, dans ces conditions, sa séparation d'avec sa meilleure amie ne peut qu'affirmer comme un destin identique dans une mer de chemins différents ou, pour le dire comme Brian Massumi, « une coprésence trans-personnelle» (Massumi, 2016), Wendy, par ce geste ultime d'amour inter-espèces, fait élever le film à la pensée affirmative de l'inégalité. Selon cette pensée qui est impliquée dans le concept même de "peuple qui manque ", toute liaison entre deux ou plusieurs termes ne peut se passer qu'en les supposant comme séparés, qu'en les affirmant dans leurs distances insurmontables. À l'opposé donc des procédés anthropomorphisants ou de résolution narrative des différences dans le cinéma classique, Reichardt met en place un véritable mécanisme de connexion, qui lie le sort de Wendy et de Lucy dans la mesure où elle nous les révèle comme deux créatures seules : seules l'une avec l'autre et seules l'une sans l'autre. Il faut remarquer que, contrairement aux autres films mineurs discutés ici, fidèles au principe bazinien de l'interdiction du montage « chaque fois qu'il est possible 
d'enfermer dans le même cadre deux éléments hétérogènes » (Daney, 1983 : 33) - par le biais de travellings, de plans fixes insistants, etc. - le montage rapide (type champcontrechamp) de la dernière scène de Wendy et Lucy semble introduire une rupture de la continuité à l'intérieur de cette brèche. Cependant la logique du montage est justifiée ici par le «thème» du grillage. En effet, ce dernier mobilise le montage tout en le reniant à l'intérieur de chaque plan (en tant qu'obstacle visuel). De cette manière, ce qui semble être une séparation "dramatique » des deux créatures, se transforme en une connexion de celles-ci en tant que séparées, solitaires, ce qui restaure la continuité interrompue, à un autre niveau, cette fois philosophique.

Ces deux types de solitudes peuvent alors être comparables au niveau de la crainte et de l'étouffement qu'ils provoquent à celles qui se retrouvent détachées ou délivrées de tout lien - pour Jacques Derrida, la phrase « je suis seul(e) avec toi », «seul(e) avec toi dans le monde » est « encore plus terrifiante, plus terriblement ambiguë que 'je suis seul(e)' (...) Tant qu'à être seul, si du moins je pouvais être seul sans toi » (Derrida, 2002-2003: 21). En même temps, ils nous aident à comprendre le rapport qu'ils désignent comme une coprésence oxymorique, qui se reflète dans le titre même du film. À cet égard, le mot « et » qui relie les noms propres «Wendy » et «Lucy » ne se contente pas d'établir une conjonction simple entre eux comme entre deux termes ou deux catégories, homme et chien, mais constitue, pour revenir à Deleuze, une multiplicité qui défait leur dualisme, de sorte qu'il n'y ait en dernière analyse ni l'un ni l'autre, mais un devenir qui les entraîne tous deux dans une " évolution non parallèle " (Deleuze \& Parnet, 1996 : 43).

21 Il y a « une sobriété, une pauvreté, une ascèse fondamentale du ET » (Deleuze \& Parnet, 1996 : 71-72), qui fait, au-delà de la littérature, le style de toute œuvre mineure, car la mise en variation ne concerne pas seulement " les éléments linguistiques, [mais aussi] les éléments non linguistiques, les variables d'expression et les variables de contenu " (Deleuze \& Guattari, 1980: 124). Ainsi pour le style mineur des films américains indépendants dont il est ici question et, plus particulièrement, de leurs scènes où la rencontre de l'homme et de l'animal ne peut se passer que dans les brèches d'un récit qui stagne. "On n'arrive à ce résultat que par sobriété, soustraction créatrice. La variation continue n'a que des lignes ascétiques» (Deleuze \& Guattari, $1980: 125)$ qui ne peuvent être tracées que par les "minorités qui travaillent la langue ", ici, chiens et gens marginaux, au fur et à mesure qu'elles ne se retrouvent pas réellement, ou qu'elles se rencontrent sans pouvoir se reconnaitre dans les conventions de celle-ci. Les exemples filmiques analysés sous la catégorie du cinéma américain mineur nous montrent combien dans le cinéma, non moins que dans les autres arts narratifs, l'impouvoir devant la langue, au sens le plus large d'une structure et d'une idéologie, peut devenir le catalyseur de tout rapprochement entre l'homme et le chien.

\section{Conclusion}

La machine anthropologique ne pourrait pas se passer d'une certaine réaction affective $\mathrm{du}$ public devant l'imagerie canine, produite du fait que, à la vue du chien, l'homme reconnaît quelque chose comme une trace d'un lien impossible avec l'animal. Le degré par lequel cette reconnaissance, en matière cinématographique, est filtrée par le logos, le langage et le sens, et ainsi construite, dépend, comme nous avons vu, du degré de sa subordination à la narration. Ce qui est important de noter, en souscrivant à 
l'argument central de Jonathan Burt, c'est que si le chien sur l'écran peut, comme tout animal, et encore plus que tout animal, être chargé de significations multiples lui donnant un statut ambigu, il est tout aussi bien un corps vulnérable et fini sur lequel les associations symboliques et métaphoriques s'écroulent, rendant possible sa rencontre avec d'autres personnages impuissants comme un peuple qui manque.

\section{BIBLIOGRAPHIE}

BAUDRILLARD, Jean (1990). La transparence du mal. Essai sur les phénomènes extrêmes. Paris: Galilée. BERGER, John (1980). About looking. London: Writers and Readers.

BORDWELL, David (2006). The way hollywood tells it. Story and Style in modern movies. Berkeley- Los Angeles - London: University of California Press.

BRODE, Douglas (2004). From Walt to Woodstock. How Disney Created the Counterculture. Austin: University of Texas Press.

BURT, Jonathan (2002). Animals in Film. London : Reaktion Books.

DANEY, Serge (1983). La rampe (Cahier critique 1970-1982). Paris : Gallimard.

DELEUZE, Gilles \& GUATTARI, Félix (1980). Capitalisme et Schizophrénie 2 : Mille Plateaux. Paris : Les Éditions de Minuit.

DELEUZE, Gilles \& PARNET, Claire (1996). Dialogues. Paris : Flammarion.

DELEUZE, Gilles (1983). Cinéma 1. L'image-mouvement. Paris : Les Éditions de Minuit.

DELEUZE, Gilles (1985). Cinéma 2. L'image-temps. Paris : Les Éditions de Minuit.

DERRIDA, Jacques (2002-2003). Séminaire. La bête et le souverain. Volume II. Paris: Galilée.

FECH, Will (2018). « "Life is Full of Difficult Decisions": Imaging Struggle in Henner Winckler's Lucy and Kelly Reichardt's Wendy and Lucy ", in Marco Abel, Jaimey Fisher (eds.). The Berlin School and Its Global Contexts: A Transnational Art Cinema. Detroit: Wayne State University Press, pp. 96-114.

FULLER-SEELEY, Kathryn \& GROSKOPF, Jeremy (2014). « The Dogs who Saved Hollywood », in Adrienne L. McLean (ed.). Dogs and Their Work in the Fiction Film. New Brunswick-New Jersey-London: Rutgers University Press, pp. 54-77.

GADD, Tim (2005). « Human-Animal Affiliation in Modern Popular Film », in Mary Sanders Pollock, Catherine Rainwater (eds.). Figuring Animals: Essays on Animal Images in Art, Literature, Philosophy, and Popular Culture. New York: Palgrave Macmillan, pp. 247-259.

GORKINKEL, Elena (2012). «Weariness, Waiting: Enduration and Art Cinema's Tired Bodies », Discourse, vol. 34, n²-3, pp. 311-347.

HARAWAY, Donna (2003). The Companion Species Manifesto: Dogs, People, and Significant Otherness. Chicago: Prickly Paradigm Press. 
HOCHSCHILD, Jennifer L. (1995). Facing Up to the American Dream. Race, Class and the Soul of the Nation. New Jersey: Princeton University Press.

MASSUMI, Brian (2016). « Vers une politique ludique de l'inclusion mutuelle » [entretien réalisé par Erik Bordeleau] [on-line]. Vacarme [N 77] (actualisé le 21/10/2016) [disponible le 03/04/2019] https://www.cairn.info/revue-vacarme-2016-4-page-84.htm

MCHUGH, Susan (2004). Dog. London: Reaktion Books.

MCHUGH, Susan (2011). Animal Stories: Narrating across Species Lines. Minneapolis-London: University of Minnesota Press.

MCLEAN, Adrienne L. (2014). «Introduction: Wonder Dogs », in Adrienne L. McLean (ed.). Dogs and Their Work in the Fiction Film. New Brunswick-New Jersey-London: Rutgers University Press, pp. 1-30.

MOLLoY, Claire (2011). Popular Media and Animals. New York: Palgrave Macmillan.

PETTMAN, Dominic (2017). Creaturely Love: How Desire Makes Us More and Less Than Human.

Minneapolis-London: University of Minnesota Press.

PICK, Anat (2011). Creaturely Poetics: Animality and Vulnerability in Literature and Film. New York: Columbia University Press.

RAYNER, Jonathan (2013). The Cinema of Michael Mann: Vice and Vindication. London-New York: Wallflower Press.

Ross, Sara \& CASTONGUAY, James (2014). « Asta the Screwball Dog: Hollywood's Canine Sidekick », in Adrienne L. McLean (ed.). Dogs and Their Work in the Fiction Film. New Brunswick-New JerseyLondon: Rutgers University Press, pp. 78-103.

SCHATZ, Thomas (1981). Hollywood Genres : Formulas, Filmmaking, and the Studio System. New York : Random House.

scotT, Anthony Oliver (2009). « Neo-Neo Realism » [on-line]. New York: The New York Times Magazine (actualisé le 17/03/2009) [disponible le 03/04/2019] <URL: https://www.nytimes.com/ 2009/03/22/magazine/22neorealism-t.html?pagewanted=all\&_r=1>

WOLF, Kelly (2014). « Promoting Lassie: The Animal Star and Constructions of 'Ideal' American Heroism » in Adrienne L. McLean (ed.). Dogs and Their Work in the Fiction Film. New Brunswick-New Jersey-London: Rutgers University Press, pp. 104-120.

ŽIzEK, Slavoj (2005). Lacrimae rerum. Paris : Éditions Amsterdam.

\section{NOTES}

1. «Les valeurs associées avec sa posture et son caractère individuels sont aussi importants pour nous les spectateurs, que l'ordre social qu'il assure. La résolution violente et le départ du héros du western à la fin du film, que ce soit dans le coucher du soleil ou dans la tombe, ne garantissent pas seulement l'ordre social mais ils perpétuent l'autonomie et la force obstinée en sa personne » (Schatz, $1981: 63$ ).

2. Deleuze écrit que le cinéma politique moderne devient mineur chaque fois qu'il « remonte en deçà de la prise de conscience " d'un peuple et que ses images ne correspondent plus «à un enchaînement d'actions, mais à des états émotionnels ou pulsionnels brisés, exprimables en visions et sons purs » (Deleuze, $1985: 286-287)$. 
3. Il convient ici de remarquer que Solondz a opté pour une pareille solution de mise-en-scène pour introduire son protagoniste canin à la fin d'une rangée de chenils.

\section{RÉSUMÉS}

Cet article propose une étude des différents procédés que le cinéma américain a employés pour poser la question de notre relation avec l'animal canin, et de la manière dont ceux-ci peuvent être ramenés à une opposition fondamentale entre, d'une part, une approche utilitariste et anthropomorphique de cette relation, inséparable d'une certaine politique de ce cinéma à l'égard du récit et de la représentation, et, d'autre part, une redéfinition de cette relation, comme quelque chose qui se passe entre les termes qu'elle relie, à savoir l'homme et le chien, sans primat de l'un sur l'autre.

This article proposes a study of the various processes which the American cinema has employed to pose the question of our relation to the canine animal, and of the way in which these processes can be brought back to a fundamental opposition between, on the one hand, a utilitarian and anthropomorphic approach, inseparable from a certain politics of this cinema with regard to narrative and representation, and, on the other hand, a redefinition of this relation, as something occurring between the terms which it connects, namely the man and the dog, and calling into question the one's primacy over the other.

\section{INDEX}

Mots-clés : cinéma mineur, chiens, rêve américain, Hollywood, impuissance

Keywords : minor cinema, dogs, American dream, Hollywood, powerlessness

\section{AUTEUR}

\section{ARGYRIOS KELERIS}

Université Paris VIII - Vincennes-Saint-Denis argyris.keleris[at]gmail.com 